\title{
CMEARTICLE
}

\section{Clinics in diagnostic imaging (187)}

Tai Jit Lenith $\underline{C h e n g}^{1,3}$, MBBS, FRCR, Yee Liang Thian ${ }^{2,3}$, MBBS, FRCR, Soon Yiew Sia ${ }^{2,3}$, MBBS, FRCR, James Thomas Patrick Decourcy $\underline{\text { Hallinan }}^{2,3}$, MBChB, FRCR
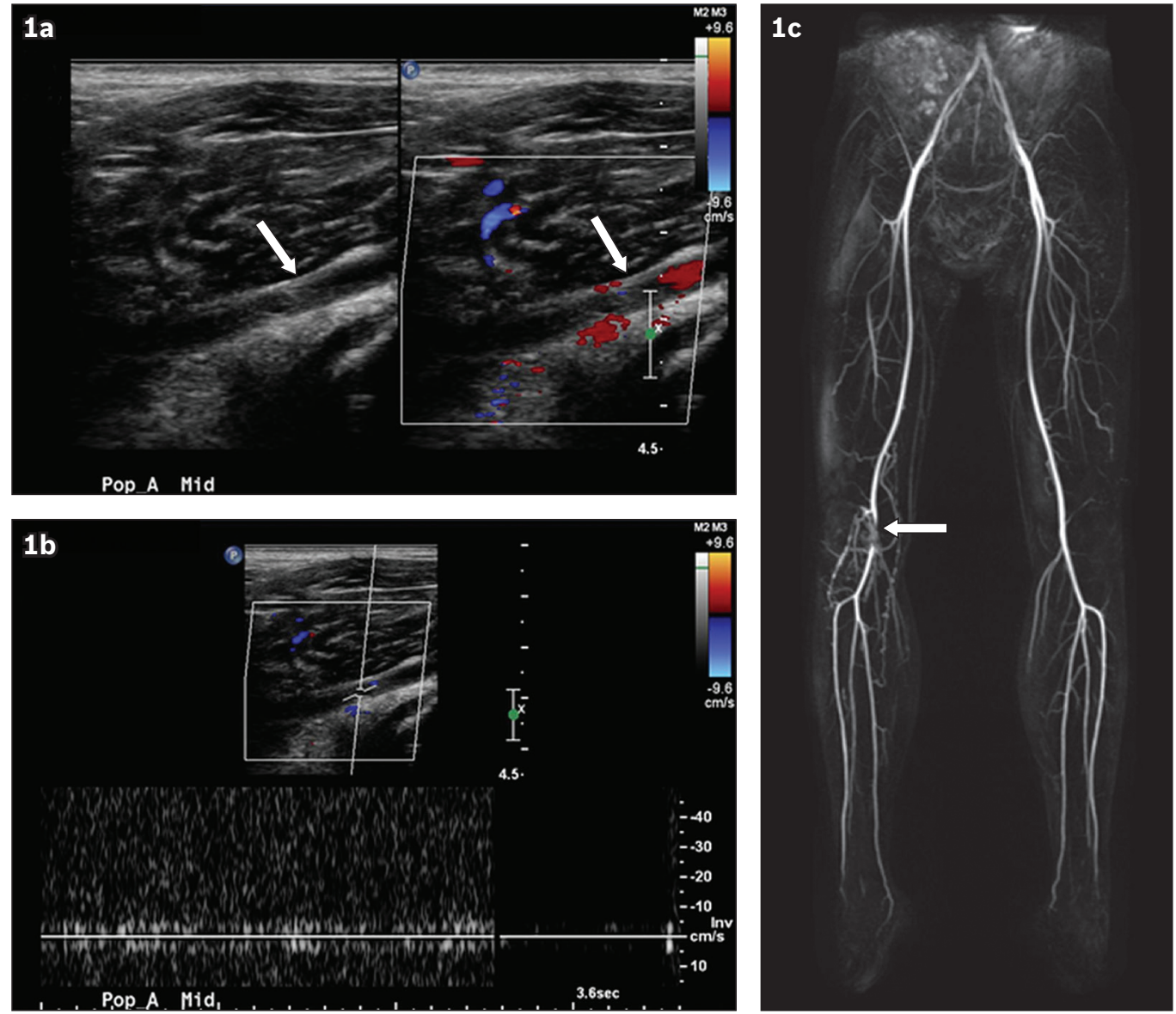

Fig. 1 (a \& b) Doppler US images of the right popliteal artery and (c) reconstructed three-dimensional MR angiogram of the right popliteal artery.

\section{CASE PRESENTATION}

A 26-year-old male patient was referred for suspected exerciseinduced claudication, which had interfered with his military duties over the past two years. He complained of right calf pain after walking approximately $200 \mathrm{~m}$, which was relieved by rest. He was an occasional smoker with no other significant cardiovascular risk factors or past medical history. Physical examination was unremarkable.

The patient underwent Doppler ultrasonography and magnetic resonance (MR) angiography of the right lower limb (Fig. 1). Additional MR imaging sequences (Fig. 2) were performed. What do the images show? What is the diagnosis?

${ }^{1}$ Department of Nuclear Medicine and Molecular Imaging, Singapore General Hospital, ${ }^{2}$ Department of Diagnostic Imaging, National University Health System, ${ }^{3} \mathrm{NUS}$ Yong Loo Lin School of Medicine, National University of Singapore, Singapore

Correspondence: Dr Cheng Tai Jit Lenith, Registrar, Department of Nuclear Medicine and Molecular Imaging, Singapore General Hospital, Outram Road, Singapore 169608. lenithcheng@mohh.com.sg 

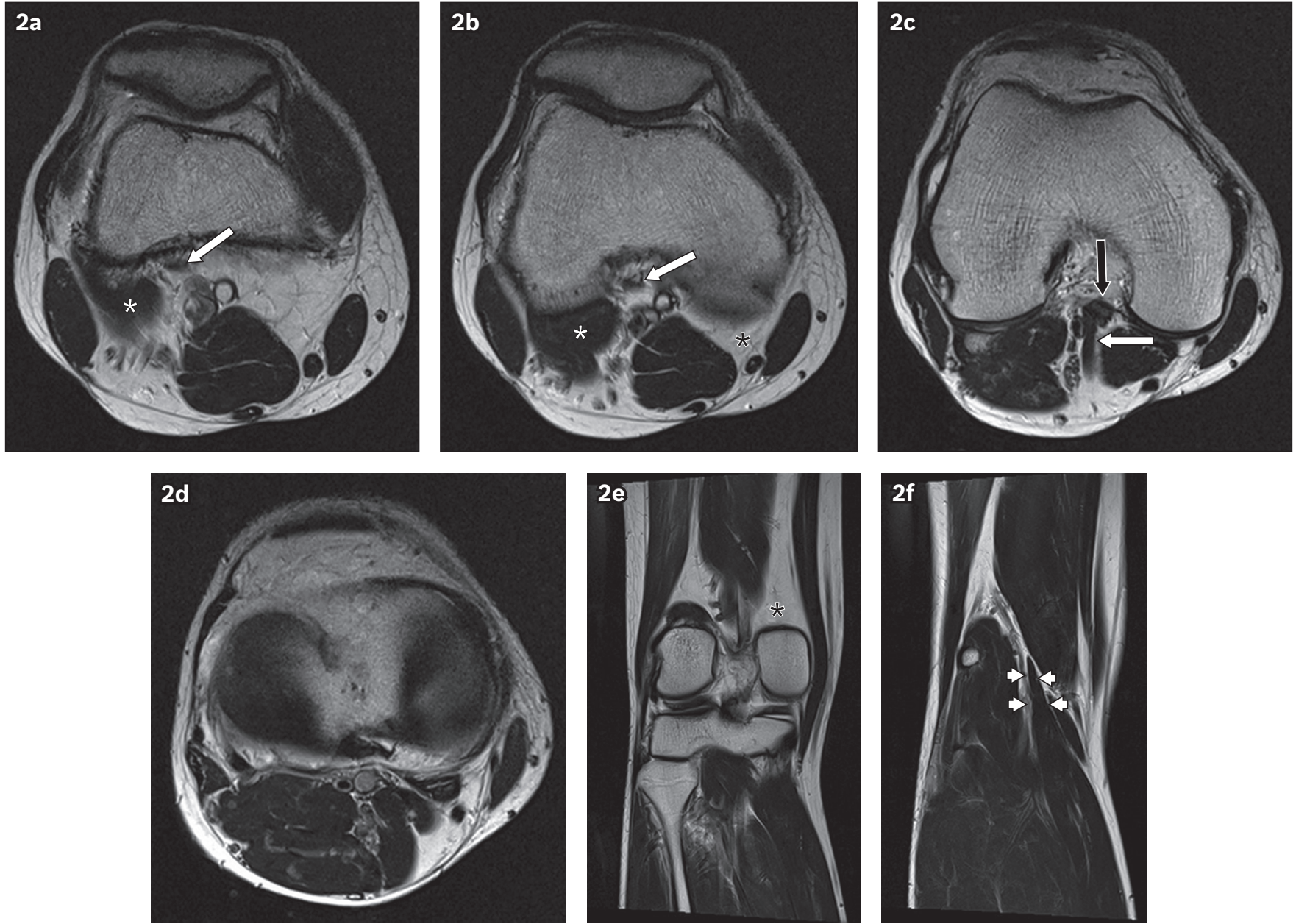

Fig. 2 Serial $(a-d)$ axial and (e \& f) coronal T2-W MR images from superior to inferior show the course of the gastrocnemius tendon.

\section{IMAGE INTERPRETATION}

Doppler ultrasonography demonstrates loss of normal arterial flow and waveform in the popliteal artery (arrow, Figs. 1a \& b). Reconstructed three-dimensional MR angiogram (Fig. 1c) shows a short segment occlusion of the right popliteal artery (arrow, Fig. 1c) with collateral formation, which suggests chronicity. No complications, such as a poststenotic aneurysm, are seen. The left-sided vessels appear to be within normal limits.

Serial axial T2-weighted (T2-W) MR images from the superior (Fig. 2a) to the inferior (Fig. 2d) aspect of the right lower limb show an anomalous origin of the medial head of the gastrocnemius tendon (white arrow, Figs. 2a-c) from the posteromedial aspect of the lateral femoral condyle. Normal origin of the lateral gastrocnemius is shown adjacent to it (white asterisk, Figs. 2a \& b). Axial T2-W (Fig. 2b) and coronal T2-W MR images (Fig. 2e) show a bare posteromedial femoral condyle (black asterisk, Figs. 2b \& e) at the expected origin of the medial head. A more posteriorly located coronal T2-W MR image (Fig. 2f) shows the medial gastrocnemius muscle (arrowheads) forming from the anomalous tendon and orientated from lateral to medial. The popliteal artery (black arrow, Fig. 2c) is compressed between the anomalous head and the posteromedial joint capsule, corresponding to the MR angiograms.

\section{DIAGNOSIS}

Popliteal artery entrapment syndrome (PAES) Type II.

\section{CLINICAL COURSE}

The patient's case was discussed at a vascular surgery and radiology multidisciplinary team meeting. In view of the patient's young age and significant clinical symptoms, surgical decompression was offered.

\section{DISCUSSION}

PAES represents an anomalous relationship between the popliteal musculotendinous structures and the artery. It is a rare but well-described cause of intermittent claudication, typically in young patients with no cardiovascular risk factors or atherosclerosis. ${ }^{(1)}$ It represents a developmental abnormality with several known variations in the popliteal fossa that leads to entrapment of the artery. PAES is a progressive condition that, if left untreated, can lead to premature arteriosclerosis and stenosis secondary to repeated chronic compression and microvascular trauma. ${ }^{(2)}$ In addition, turbulent flow through a tight stenosis can lead to poststenotic aneurysm formation. Hence, early diagnosis or exclusion of PAES is of paramount importance in a young adult presenting with intermittent claudication. ${ }^{3}$ 

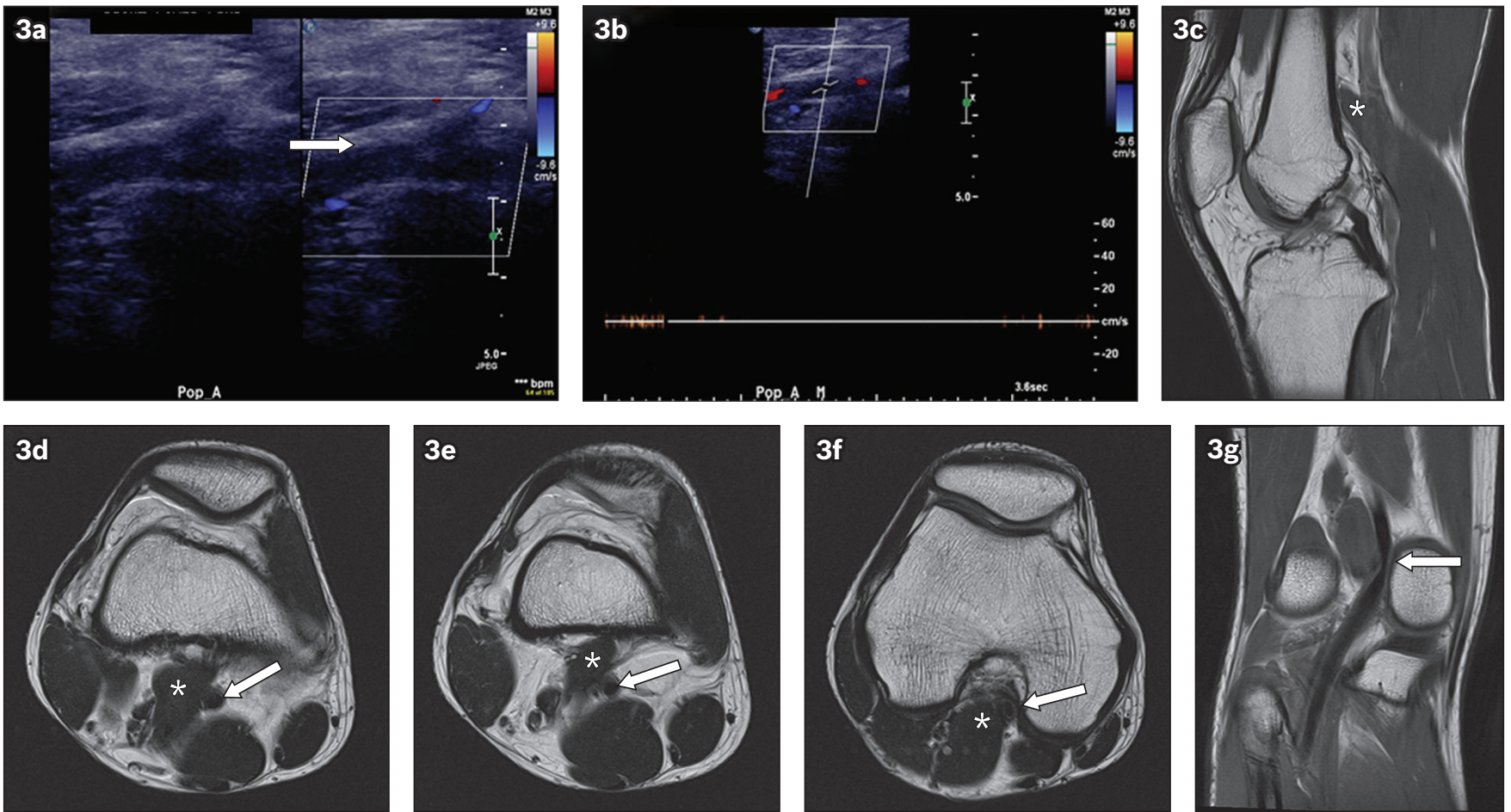

Fig. 3 A 23-year-old female patient presented with exercise-induced claudication of the right lower limb. (a \& b) Doppler US images of the right knee show loss of normal arterial flow and waveform in the right popliteal artery. (c) Sagittal T2-W MR image shows the normal origin of the medial head of the gastrocneumius muscle (white asterisk in $3 c$ ). ( $d-f$ ) Axial T2-W MR images show an aberrant medial course of the popliteal artery (white arrows in Figs. 3d-f) compressed by the adjacent medial head of the gastrocnemius muscle (white asterisk in Figs. 3d-f). (g) Coronal T2-W MR image shows the focal entrapment of the popliteal artery (white arrow). The patient was diagnosed with PAES Type I.

PAES has a reported clinical prevalence of approximately $0.17 \%$ in young males entering military service, although the true incidence may be much higher, with anatomical PAES having a reported prevalence of $3.5 \%$ in autopsy cases. ${ }^{(4,5)}$ PAES can also occur bilaterally in approximately $27 \%-67 \%$ of affected patients. ${ }^{(6)}$ Several classification systems exist, one of which is a widely used classification system proposed by Love and Whelan in 1965 that classified PAES into six main subtypes (Box 1).

PAES Types I-V involve an abnormal relationship between the vessels, musculotendinous structures or fibrous bands.(7) Fig. 3 shows PAES Type I in a different patient, with an aberrant medial course of the popliteal artery that is entrapped by a normally positioned medial head of the gastrocnemius muscle. PAES Type VI is a functional form of PAES in which the popliteal artery and medial head of the gastrocnemius are both in normal anatomical positions, but the popliteal artery is entrapped by a hypertrophied gastrocnemius muscle. ${ }^{(8)}$

For patients with suspected PAES, there is no widely accepted consensus for the ideal diagnostic workup. In our centre, we used Doppler ultrasonography for the initial evaluation of the popliteal artery, followed by MR angiography/imaging for a more detailed evaluation. MR imaging has several advantages. (a) There is no radiation exposure for the patient compared with computed tomography (CT) and conventional digital subtraction angiography (DSA). (b) MR imaging has superior soft tissue contrast to clearly delineate any aberrant popliteal fossa anatomy. This will help guide any surgical intervention. (c) Complications such as poststenotic aneurysm formation can be readily identified. (d) It can differentiate PAES from other differential diagnoses,

\begin{tabular}{|c|c|}
\hline 1 & $\begin{array}{l}\text { Aberrant medial course of the popliteal artery, } \\
\text { compressed by normal medial head of the gastrocnemius }\end{array}$ \\
\hline II & $\begin{array}{l}\text { Normal course of the popliteal artery, compressed by } \\
\text { an aberrant lateral origin of the medial head of the } \\
\text { gastrocnemius muscle }\end{array}$ \\
\hline III & $\begin{array}{l}\text { Normal course of the popliteal artery, surrounded } \\
\text { and compressed by an accessory head of the medial } \\
\text { gastrocnemius muscle }\end{array}$ \\
\hline IV & $\begin{array}{l}\text { Popliteal artery lying deep in the popliteal fossa and } \\
\text { compressed by the popliteus muscle or a fibrous band }\end{array}$ \\
\hline V & Any of the above with involvement of the popliteal vein \\
\hline VI & $\begin{array}{l}\text { Normal anatomical positions of the popliteal artery, } \\
\text { compressed by a hypertrophied medial head of } \\
\text { gastrocnemius muscle }\end{array}$ \\
\hline
\end{tabular}

including vasculitis or cystic adventitial disease, which are also rare vascular (predominantly arterial) pathologies that occur in young adults with no cardiovascular risk factors.

Our MR angiography/imaging protocol utilises gadoliniumenhanced MR angiography of both lower limbs from the common femoral arteries to the distal leg. This is performed at rest in a neutral position. Non-contrast time-of-flight images are another option but, in our experience, are not as robust as contrast-enhanced images and more prone to motion artefacts. Anatomical T2-W axial and coronal images are then acquired to demonstrate the origins and alignment of the medial and lateral heads of the gastrocnemius and popliteus muscles and their associations with the popliteal artery, vein and nerves. These images are acquired at rest. If vascular compression is 

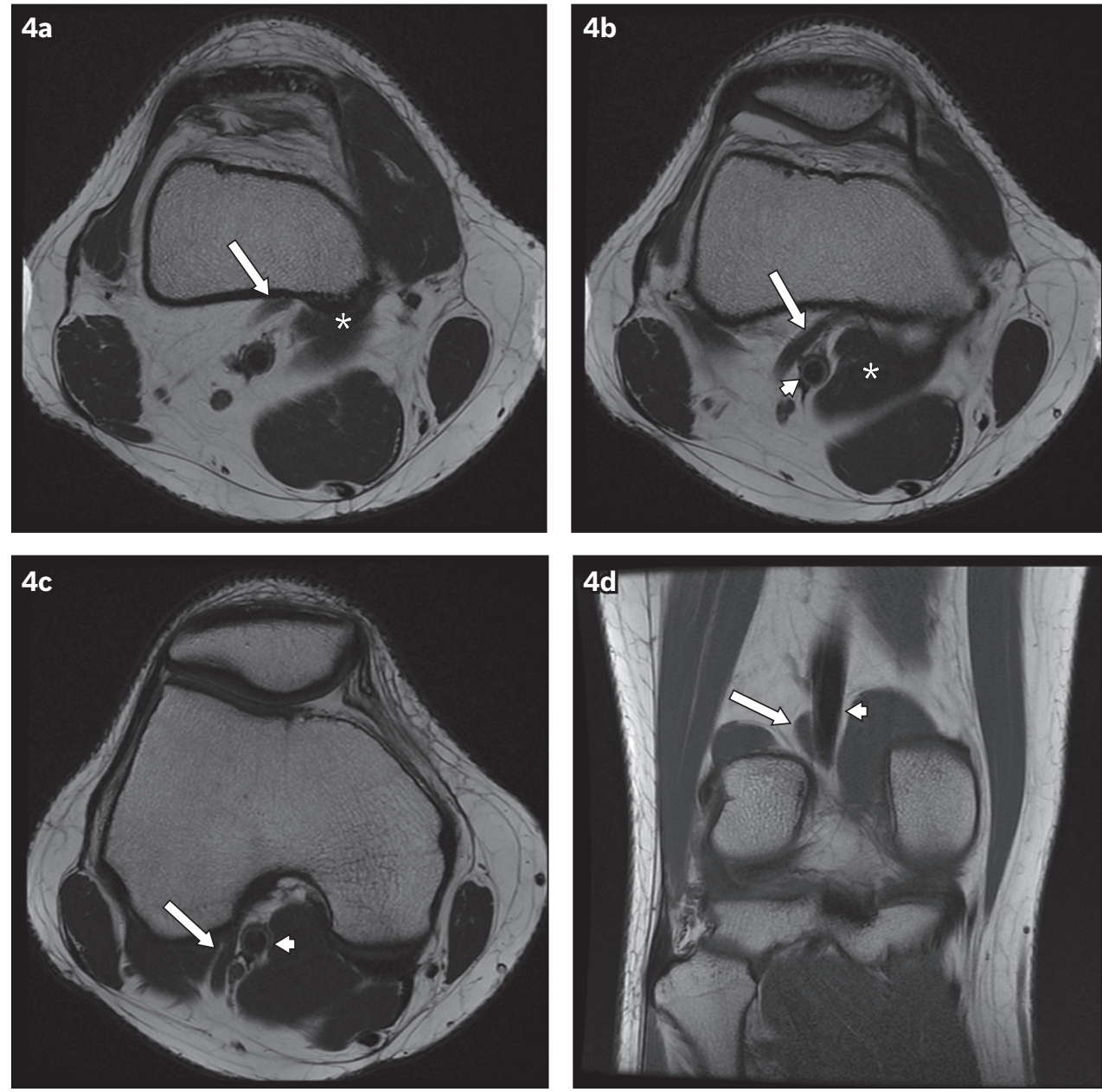

Fig. 4 Incidental finding of aberrant popliteal fossa anatomy with no entrapment of the popliteal artery. (a-c) Axial T2-W and (d) coronal T1-W MR images show an accessory head of the medial gastrocnemius muscle (arrow in Figs. 4a-d) surrounding the popliteal artery (arrowheads in Figs. 4b-d). The normal medial head of the gastrocnemius muscle is also clearly shown (asterisk in Figs. $4 a$ \& b).

not observed, additional T2-W two-dimensional steady state images are acquired across the popliteal fossa using provocative manoeuvres. Patients are instructed to perform continuous plantar flexion and dorsiflexion of the feet to recreate their claudication symptoms, after which they maintain plantar flexion while the scan is performed. This may help to accentuate any vascular compression. The main problem with this imaging method is the difficulty in maintaining the forced plantar flexion due to patient discomfort and exhaustion, which may result in motion artefacts that degrade the image quality.

Aberrant anatomy in the popliteal fossa may be incidentally diagnosed on MR imaging in asymptomatic patients (Fig. 4). For this reason, correlation between the clinical history of intermittent claudication and imaging features of PAES is vital for accurate diagnosis. Other options for the diagnosis of PAES include CT angiography and DSA. Both modalities demonstrate the vascular anatomy in high detail. DSA also has the ability to dynamically evaluate for vessel compression/occlusion using provocative manoeuvres. However, both modalities, especially DSA, cannot accurately evaluate the musculotendinous anatomy of the popliteal fossa compared to MR imaging. In addition, they involve exposure to radiation, and DSA is an invasive procedure.

Potential differential diagnoses of PAES in young patients without vascular risk factors include cystic adventitial disease


Fig. 5 A 26-year-old man presented with chronic left lower limb claudication. (a) Reconstructed MR angiogram of both lower limbs shows abrupt truncation of flow-related signal in the left popliteal artery (arrow).

(b) Coronal T1-W image shows severe stenosis and mural thickening of a segment of the popliteal artery (arrowheads) with no evidence of popliteal artery entrapment.

and other forms of vasculitis. Fig. 5 shows severe stenosis of the popliteal artery with no evidence of popliteal artery entrapment.

Treatment for PAES involves a multidisciplinary surgical approach and depends on the degree of vascular compromise and the type of compression. The primary aim is to relieve the 
compression of the artery using musculotendinous section, restoring normal arterial flow to the limb. ${ }^{(9)}$ Chronic occlusion and arteriosclerosis of the vessels may require additional vascular bypass or grafting, which commonly utilise the saphenous vein. ${ }^{(10)}$ Endovascular repair alone is not recommended due to a high risk of re-occlusion, as the underlying cause of popliteal entrapment has not been addressed.

In conclusion, PAES is a rare cause of intermittent claudication or acute limb ischaemia that is especially relevant

ABSTRACT A 26-year-old male patient was referred for exercise-induced claudication that had interfered with his military duties for the past two years. He was an occasional smoker with no other significant cardiovascular risk factors. Initial Doppler ultrasonography showed narrowing of the popliteal artery. Further evaluation with magnetic resonance angiography demonstrated a short segment occlusion of the popliteal artery secondary to an anomalous origin of the medial head of the gastrocnemius muscle from the lateral femoral condyle. Based on the clinical presentation and imaging findings, he was diagnosed with popliteal artery entrapment syndrome. In this article, we discussed the typical presenting signs and symptoms of popliteal artery entrapment syndrome, the different imaging modalities available to aid diagnosis, classification of the condition and the available treatment options.

Keywords: claudication, MRA, MRI, popliteal artery, vascular in young adults with no cardiovascular risk factors. Early diagnosis and treatment are important to prevent complications of this progressive condition. Radiologists play an essential role in the diagnosis of PAES. MR angiography/ imaging can provide noninvasive detection of vascular stenosis, as well as an accurate assessment of aberrant popliteal fossa anatomy.

\section{REFERENCES}

1. Sinha S, Houghton J, Holt PJ, et al. Popliteal entrapment syndrome. J Vasc Surg 2012; 55:252-262.e30.

2. Williams C, Kennedy D, Bastian-Jordan M, et al. A new diagnostic approach to popliteal artery entrapment syndrome. J Med Radiat Sci 2015; 62:226-9.

3. Gokkus K, Sagtas E, Bakalim T, et al. Popliteal entrapment syndrome. A systematic review of the literature and case presentation. Muscles Ligaments Tendons J 2014; 4:141-8.

4. Bouhoutsos J, Daskalakis E. Muscular abnormalities affecting the popliteal vessels. Br J Surg 1981; 68:501-6.

5. Gibson MH, Mills JG, Johnson GE, Downs AR. Popliteal entrapment syndrome. Ann Surg 1977; 185:341-8.

6. Hai Z, Guangrui S, Yuan Z, et al. CT angiography and MRI in patients with popliteal artery entrapment syndrome. AJR Am J Roentgenol 2008; 191:1760-6.

7. Soobrah R, Nawaz A, Hussain T. Popliteal artery entrapment syndrome presenting with acute limb ischaemia: a case report. Case Rep Med 2010; 2010:281925.

8. Hislop M, Kennedy D, Cramp B, Dhupelia S. Functional popliteal artery entrapment syndrome: poorly understood and frequently missed? A review of clinical features, appropriate investigations, and treatment options. J Sports Med (Hindawi Publ Corp) 2014; 2014:105953.

9. Gourgiotis S, Aggelakas J, Salemis N, Elias C, Georgiou C. Diagnosis and surgical approach of popliteal artery entrapment syndrome: a retrospective study. Vasc Health Risk Manag 2008; 4:83-8.

10. Kim SY, Min SK, Ahn S, et al. Long-term outcomes after revascularization for advanced popliteal artery entrapment syndrome with segmental arterial occlusion. J Vasc Surg 2012; 55:90-7. 


\section{SINGAPORE MEDICAL COUNCIL CATEGORY 3B CME PROGRAMME} (Code SMJ 201806B)

Question 1. Regarding popliteal artery entrapment syndrome (PAES):

(a) It occurs predominantly in the elderly age group.

(b) PAES is a progressive condition that, if left untreated, can lead to premature arteriosclerosis and stenosis.

(c) It can occur in both lower limbs.

(d) Turbulent flow through a tight arterial stenosis can lead to poststenotic aneurysm formation.

Question 2. Regarding PAES:

(a) An accessory medial gastrocnemius leads to entrapment in Type III PAES.

(b) It always occurs due to an aberrant origin of the gastrocnemius muscle.

(c) It can occur in patients without aberrant anatomy.

(d) It typically occurs in patients without cardiovascular risk factors.

Question 3. Regarding ultrasonography in PAES:

(a) It is commonly used as the initial screening tool in those with intermittent claudication and suspected PAES.

(b) Ultrasonography alone is sufficient for diagnosing PAES.

(c) Ultrasonography is the preferred modality due to high anatomical detail.

(d) Ultrasonography is the only modality with which the radiologist can dynamically evaluate for vessel compression with provocative manoeuvres.

Question 4. Regarding computed tomography $(\mathrm{CT})$ /magnetic resonance (MR) imaging in PAES:

(a) It is performed in all patients who report symptoms of claudication.

(b) CT is superior to MR imaging in the assessment of the underlying anatomical causes of PAES.

(c) CT is the preferred modality to exclude other causes of claudication such as vasculitis or cystic adventitial disease.

(d) MR imaging is the preferred modality due to its reduced susceptibility to motion artefacts as compared to CT.

Question 5. Regarding the clinical management of PAES:

(a) Endovascular treatment has equal outcomes with surgical intervention.

(b) The main goal of treatment is to relieve arterial compression.

(b) A saphenous vein graft is often used when bypass or grafting is required.

(c) Re-occlusion is a possible complication after treatment.



\section{Doctor's particulars:}

Name in full:

MCR no:

Specialty:

Email:

\section{SUBMISSION INSTRUCTIONS}

Visit the SMJ website: http://www.smj.org.sg/current-issue and select the appropriate quiz. You will be redirected to the SMA login page.

For SMA member: (1) Log in with your username and password (if you do not know your password, please click on 'Forgot your password?'). (2) Select your answers for each quiz and click 'Submit'.

For non-SMA member: (1) Create an SMJ CME account, or log in with your SMJ CME username and password (for returning users). (2) Make payment of SGD 21.40 (inclusive of $7 \%$ GST) via PayPal to access this month's quizzes. (3) Select your answers for each quiz and click 'Submit'.

RESULTS:

(1) Answers will be published online in the SMJ August 2018 issue. (2) The MCR numbers of successful candidates will be posted online at the SMJ website by 6 August 2018. (3) Passing mark is $60 \%$. No mark will be deducted for incorrect answers. (4) The SMJ editorial office will submit the list of successful candidates to the Singapore Medical Council. (5) One CME point is awarded for successful candidates. (6) SMC credits CME points according to the month of publication of the CME article (i.e. points awarded for a quiz published in the December 2017 issue will be credited for the month of December 2017, even if the deadline is in January 2018).

Deadline for submission: (June 2018 SMJ 3B CME programme): 12 noon, 30 July 2018. 